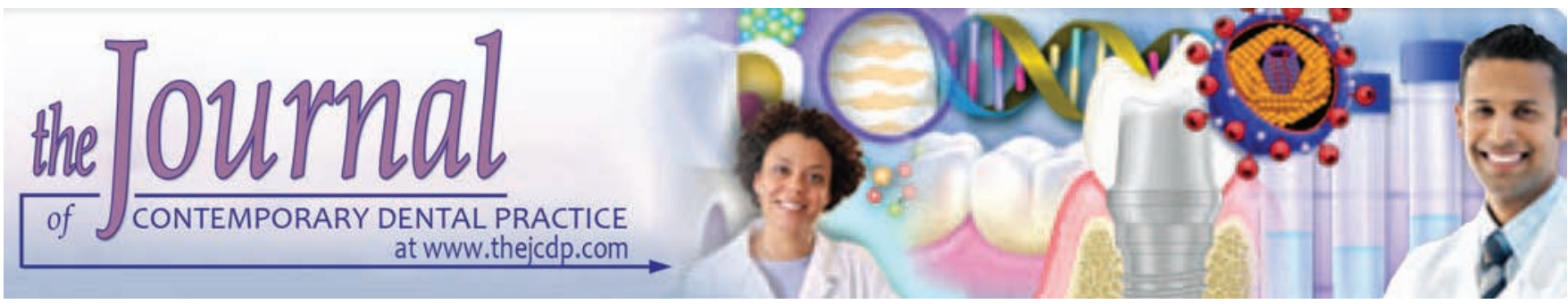

\title{
An Alternative Approach for Space Opening in a Bilateral Maxillary Lateral Incisor Agenesis Patient using Miniplates
}

\author{
${ }^{1}$ Gladistone C Meros, ${ }^{2}$ Aline Shoji, ${ }^{3}$ Selly Suzuki, ${ }^{4}$ Luiz R Paranhos, ${ }^{5}$ Rodrigo Manfroi \\ ${ }^{6}$ Jonathas Claus, ${ }^{7}$ Aguinaldo Garcez
}

\begin{abstract}
Aim: This case report aimed to present an orthodontic mechanic alternative for space opening in a patient missing maxillary lateral incisors, using miniplates as anchorage, associated with self-ligating brackets.
\end{abstract}

Background: Dental agenesis affects the population causing esthetic and functional damages. The prevalence of missing maxillary lateral incisors should be considered for its significant rate and negative impact on smile esthetics.

Case report: This treatment was chosen based on the presence of balanced facial pattern, large canine anatomy, the need to improve dental occlusion to prevent further wear, and patient's esthetic complaint. To obtain the results, upper third molars were extracted, and two miniplates were installed to distalize the upper arch with no need for patient compliance or auxiliary devices. Self-ligating brackets were used to reduce friction on posterior teeth, thus facilitating movement with light force application. At the end of 19 months, the patient presented with class I good overbite and overjet, and adequate space for implant and prosthetics; also, good facial esthetic was maintained.

Conclusion: When indicated, space opening may provide excellent esthetics and functional results, and even more predictable results when skeletal anchorage miniplates are used to distalize all posterior teeth.

Clinical significance: Considering the high level of esthetic and functional compromise caused by dental agenesis, the

1-3,7Department of Orthodontics, São Leopoldo Mandic Dental School and Research Center, Campinas, São Paulo, Brazil

${ }^{4}$ Department of Dentistry, Federal University of Sergipe, Lagarto Sergipe, Brazil

${ }^{5}$ Department of Prosthesis, São Leopoldo Mandic Dental School and Research Center, Campinas, São Paulo, Brazil

${ }^{6}$ Department of Oral Surgery, Federal University of Santa Catarina, Florianópolis, Santa Catarina, Brazil

Corresponding Author: Gladistone C Meros, Department of Orthodontics, São Leopoldo Mandic Dental School and Research Center, Campinas, São Paulo, Brazil, Phone: +554699117799 e-mail: gladistone.fb@gmail.com technique hereby described represents a viable mechanic alternative within orthodontic possibilities.

Keywords: Anchorage, Case report, Distalization, Missing teeth.

How to cite this article: Meros GC, ShojiA, Suzuki S, Paranhos LR, Manfroi R, Claus J, Garcez A. An Alternative Approach for Space Opening in a Bilateral Maxillary Lateral Incisor Agenesis Patient using Miniplates. J Contemp Dent Pract 2017;18(12):1198-1205.

Source of support: Nil

Conflict of interest: None

\section{BACKGROUND}

Dental agenesis is a common developmental anomaly that consists of a congenital absence of one or more teeth, and in permanent dentition, the prevalence ranges from 2.7 to $11.3 \%$ (not including third molars). ${ }^{1}$ Missing maxillary lateral incisors represent $20 \%$ of the overall dental anomalies, ${ }^{2}$ and, in permanent dentition, the prevalence ranges between 0.8 and $2 \%$, with bilateral agenesis occurring more frequently than unilateral agenesis. ${ }^{1}$

There are at least two options for treating missing lateral incisors: Space opening followed by a prosthetic solution or space closure with mesial repositioning of the canine and a set of reanatomization procedures, including on premolars. Each of the available treatments has its own advantages, disadvantages, indications, and limitations, such as patient age, anatomy of upper canines, type of malocclusion, space available for prosthetic placement, and patient's esthetic complaints. ${ }^{3,4}$

Some advantages of space closure are improved periodontal conditions, lower cost, the possibility to be performed along the skeletal growth of patients, and the completion of overall treatment at the end of orthodontic treatment. 5,6 There are also some disadvantages, as follows: Difficult retention, because spaces tend to reopen in the maxillary anterior region after closure; potentially compromised functional occlusion, considering that a 

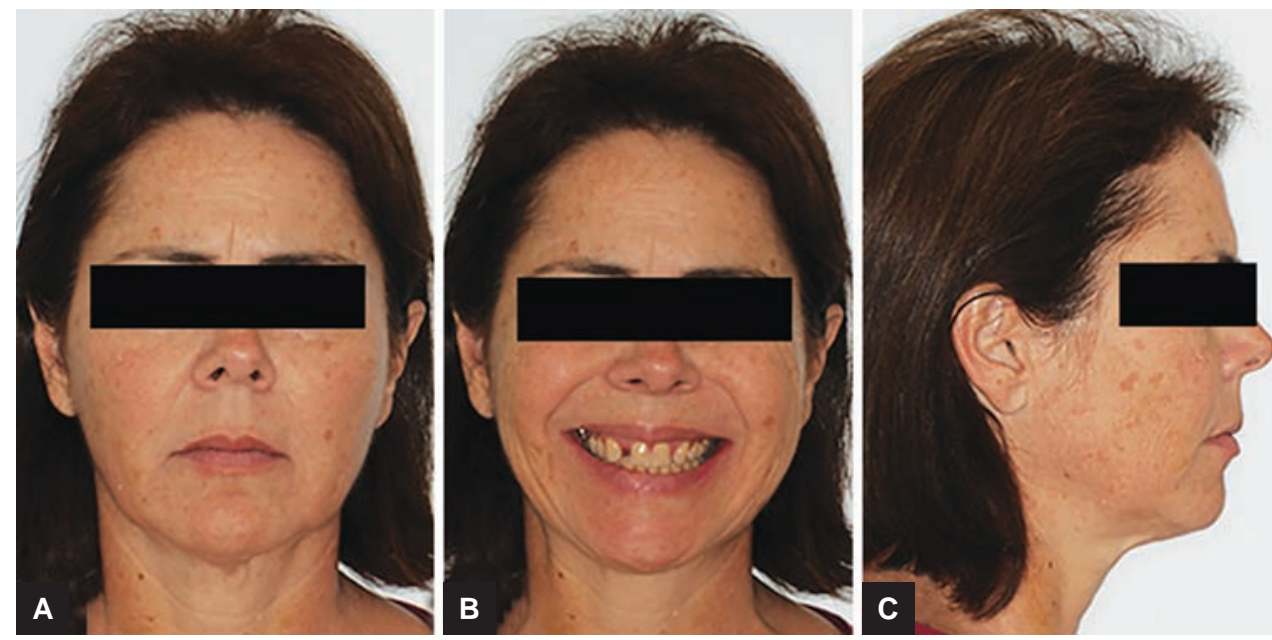

Figs $1 \mathrm{~A}$ to $\mathrm{C}$ : Facial and intraoral images before treatment

cuspid-protected functional occlusion is generally not feasible; lateral group guidance, usually achieved on maxillary bicuspids and relocated cuspids; potential abrasion on mandibular lateral incisors in case of excessive contact with the maxillary cuspid; presence of undesirable buccal corridors from the reduction in arch circumference; and esthetic success depending on the clinicians' ability to reanatomize canines and premolars. ${ }^{3,6}$ On the contrary, advantages of orthodontic space reopening are a more conservative approach (preserving the morphological features of canines and first premolars), preservation of ideal intercuspation, promotion of canine-protected occlusion, and maintenance of a harmonic profile. ${ }^{2}$ Disadvantages include the accumulation of plaque and gingivitis around implants; an esthetic issue involving tooth shade and transparency along with gingival color, contour, and margin levels; and the usual prevention of implant placement until all skeletal growth is completed and tooth eruption has ceased.?

The professional decision for space opening implies the end of craniofacial growth, especially for an implantsupported prosthesis. ${ }^{7}$ Over the past few years, the increase of skeletal anchorage use has made space opening a more feasible option, for it does not compromise canine functional occlusion.

Upper molar distalization using miniplates may offer efficient skeletal anchorage and predictability for the treatment of class II molar relationship, and it does not require patient compliance. According to De Clerck and Swennen, the overall success rate of miniplate anchorage regarding maxillary stability is approximately $97 \%$. Thus, this study describes the treatment of class II patient missing lateral incisors bilaterally, using miniplates for distalization.

\section{CASE REPORT}

Female patient, DC, Caucasian, 51 years and 6 months old, was referred to the orthodontic clinic complaining of "tooth wearing and unesthetic smile" (Fig. 1). The patient presented good general and oral health, history of cavities that were satisfactorily treated, and no temporomandibular joint disorders. Missing both right and left lateral incisors was the main issue (\#22 and \#12).

According to facial examination and cephalometric measurements of the lateral X-ray, the patient had normal skeletal patterns, no asymmetry, straight profile, and good position of upper and lower incisors (Table 1). Intraoral examination revealed the presence of $3.8-\mathrm{mm}$ space between the right upper central incisor and the canine and 2-mm space between the left upper central incisor and the canine, due to missing lateral incisors bilaterally.

Table 1: Baseline and posttreatment measurements obtained in the cephalometric analysis

\begin{tabular}{llll}
\hline Factor & Clinical norm & Before treatment & After treatment \\
\hline NAP & $0^{\circ}$ & $7.61^{\circ}$ & $7.76^{\circ}$ \\
SNA & $82^{\circ}$ & $83.83^{\circ}$ & $83.24^{\circ}$ \\
SNB & $80^{\circ}$ & $79.55^{\circ}$ & $78.62^{\circ}$ \\
ANB & $2^{\circ}$ & $4.28^{\circ}$ & $4.62^{\circ}$ \\
SDN & $76^{\circ}$ & $75.87^{\circ}$ & $75.19^{\circ}$ \\
SN.GoGn & $32^{\circ}$ & $31.95^{\circ}$ & $32.33^{\circ}$ \\
SN.Gn & $66^{\circ}$ & $68.36^{\circ}$ & $69.50^{\circ}$ \\
1.1 & $131^{\circ}$ & $134.22^{\circ}$ & $128.50^{\circ}$ \\
1.NA & $22^{\circ}$ & $12.62^{\circ}$ & $11.47^{\circ}$ \\
1-NA & $4 \mathrm{~mm}$ & $3.62 \mathrm{~mm}$ & $5.02 \mathrm{~mm}$ \\
1.NB & $25^{\circ}$ & $28.88^{\circ}$ & $35.42^{\circ}$ \\
1-NB & $4 \mathrm{~mm}$ & $6.86 \mathrm{~mm}$ & $6.92 \mathrm{~mm}$ \\
1-Line I & 0 & $-3.69 \mathrm{~mm}$ & $-4.27 \mathrm{~mm}$ \\
U6-PTV & $21 \mathrm{~mm}$ & $19.52 \mathrm{~mm}$ & $16.37 \mathrm{~mm}$ \\
H-nose & $9-11 \mathrm{~mm}$ & $8.13 \mathrm{~mm}$ & $6.79 \mathrm{~mm}$ \\
FMA & $25^{\circ}$ & $27.63^{\circ}$ & $23.52^{\circ}$ \\
FMIA & $68^{\circ}$ & $54.99^{\circ}$ & $52.02^{\circ}$ \\
IMPA & $87^{\circ}$ & $97.38^{\circ}$ & $104.46^{\circ}$ \\
\hline
\end{tabular}

FMA: Frankfort mandibular plane angle; FMIA: Frankfort mandibular incisor axis angle; IMPA: Incisor mandibular plane angle; SNB: Sella nasion point B angle; SNA: Sella nasion point A angle; ANB: A point, nasion B point angle; NA: Nasion point A; NB: Nasion point B; GoGn: Gonion gnathion 

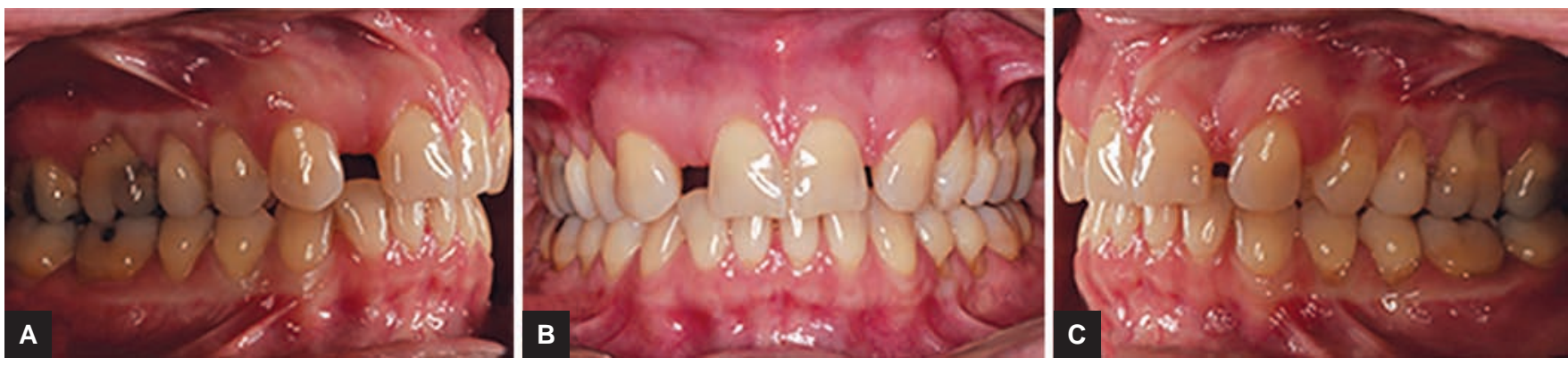

Figs 2A to C: Intraoral images before treatment: (A) Right side; (B) front; and (C) left side
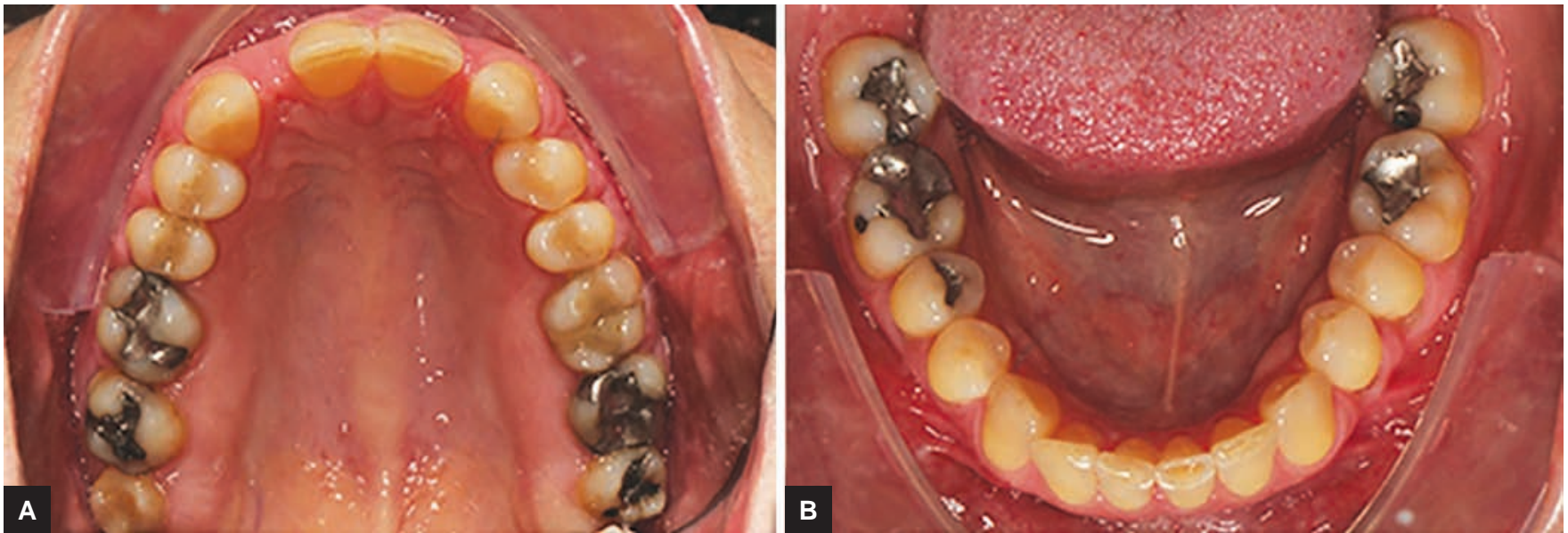

Figs 3A and B: Intraoral images before treatment: (A) Upper occlusal; and (B) lower occlusal
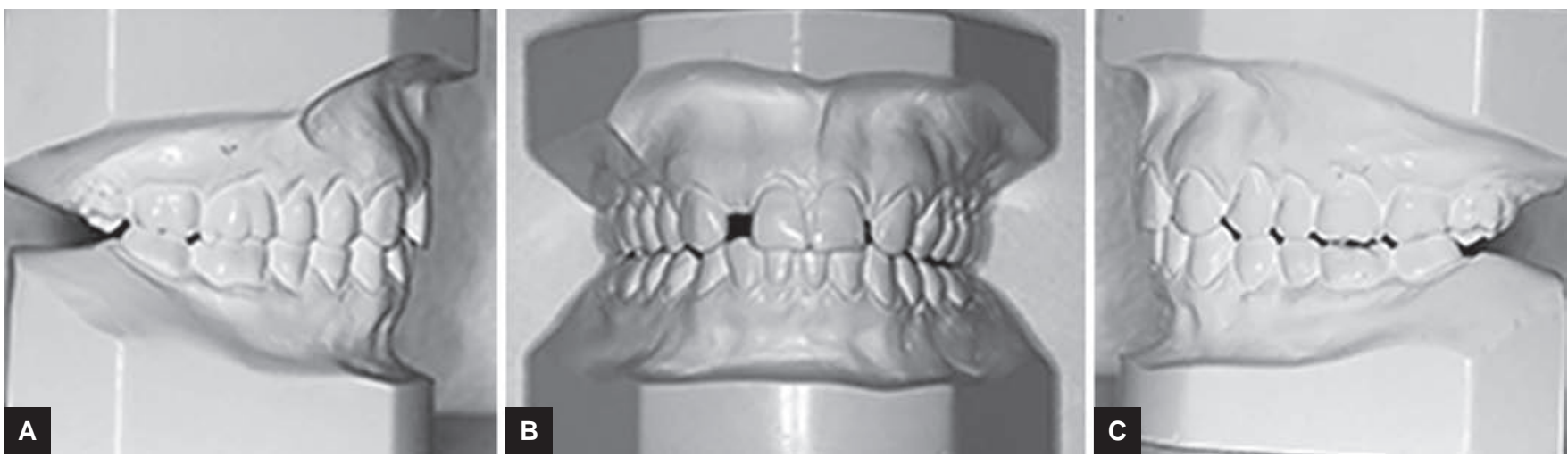

Figs 4A to C: Plaster casts before treatment

Posterior malocclusion showed class II, division 1 malocclusion $(3.5 \mathrm{~mm})$ on both sides, and $1.5 \mathrm{~mm}$ of the upper midline toward the left side (Figs 1 to 4 ).

The radiographic examinations showed the presence of erupted upper third molars only. No other abnormality was verified (Fig. 5).

The treatment goals of this case report consisted of creating a class I occlusion with bilateral canine excursion and anterior guidance, opening spaces on both sides to obtain conditions for implants and maintain a harmonic profile.

An alternative approach would have treated this malocclusion by closing the spaces with mesial drifting of posterior teeth and canine anchoring by miniscrews and

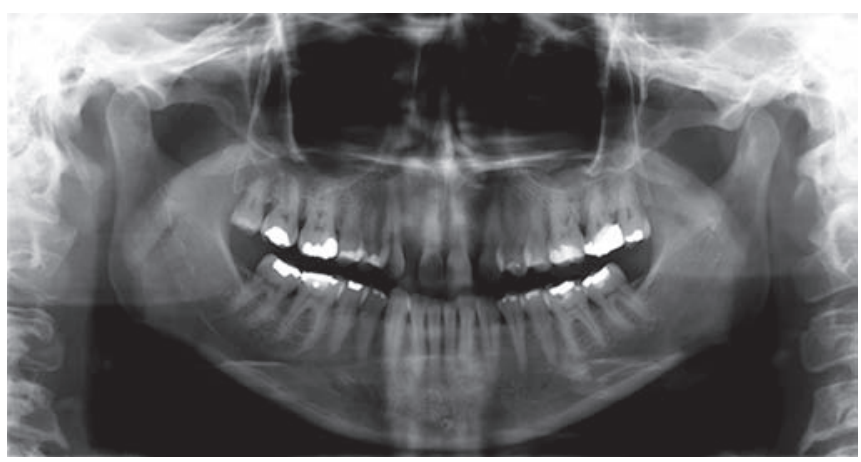

Fig. 5: Panoramic radiograph before treatment showing absence of both upper lateral incisors and presence of both upper wisdom teeth 


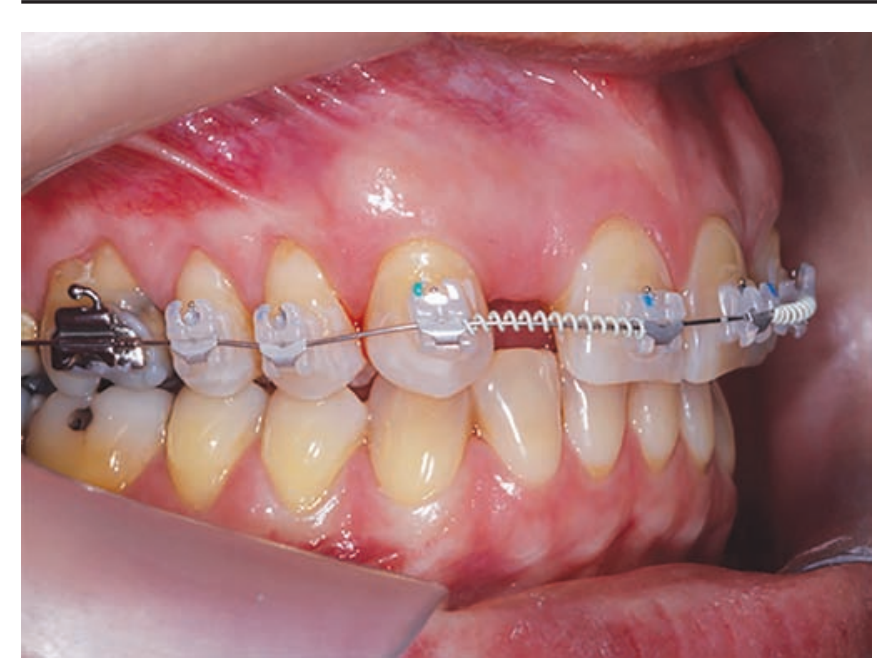

Fig. 6: Self-ligating esthetic braces system

reanatomization steps (canines turning into lateral incisors, and premolars turning into canines). Although this option was considered, the spaces tend to reopen during retention, which may compromise functional occlusion.

\section{Treatment Progress}

To obtain enough space opening, the treatment plan included the distalization of posterior teeth using miniplates as anchorage after extracting both the right and left upper third molars.

The orthodontic treatment began with the complete bonding of the fixed self-ligating appliance, Roth prescription (In-Ovation ${ }^{\mathrm{TM}} \mathrm{C}$; GAC) with $0.022^{\prime \prime} \times 0.028^{\prime \prime}$ slot
(Fig. 6). For initial alignment and leveling, Sentalloy 0.016" Medium Upper Standard Arch Form Medium (GAC) was used, and later, Accu-Force G3 Square 0.020" × 0.020" Upper AccuForm Medium (GAC) was used.

Two skeletal anchorage miniplates were inserted between the upper first molar and the upper second molar, on both sides (Fig. 7). A full-thickness mucoperiosteal flap was reflected buccally under local anesthesia to expose the zygomatic crest. The 3-hole Y-type titanium miniplates (Signo Vinces, Curitiba, PR, Brazil) were placed in the buccal cortical plate of the maxilla and secured by three monocortical screws above the dental roots.

About 3 weeks after surgery, as recommended by De Clerck, a 150-g force was applied from the miniplates to soldered hooks on the archwire; sliding jigs were also used to obtain a distal displacement of the upper molars (Fig. 7). Two temporary teeth with lateral incisor brackets bonded on them were placed over the orthodontic wire to camouflage the space opening and improve patient esthetics. In addition, during the distal movement, acrylic resin increments were added to both sides of the temporary crown to increase the width and improve esthetics. No mobility of the anchorage miniplates was observed during treatment.

To finish upper arch distalization and gain intercuspation, power chain elastics attached from miniplates to canine brackets were used, as well as class II elastics (Fig. 8).

After 12 months of active treatment, spaces created for lateral incisor implants were measured using computed tomographic images, which revealed enough space and favorable bone thickness and height, allowing implant
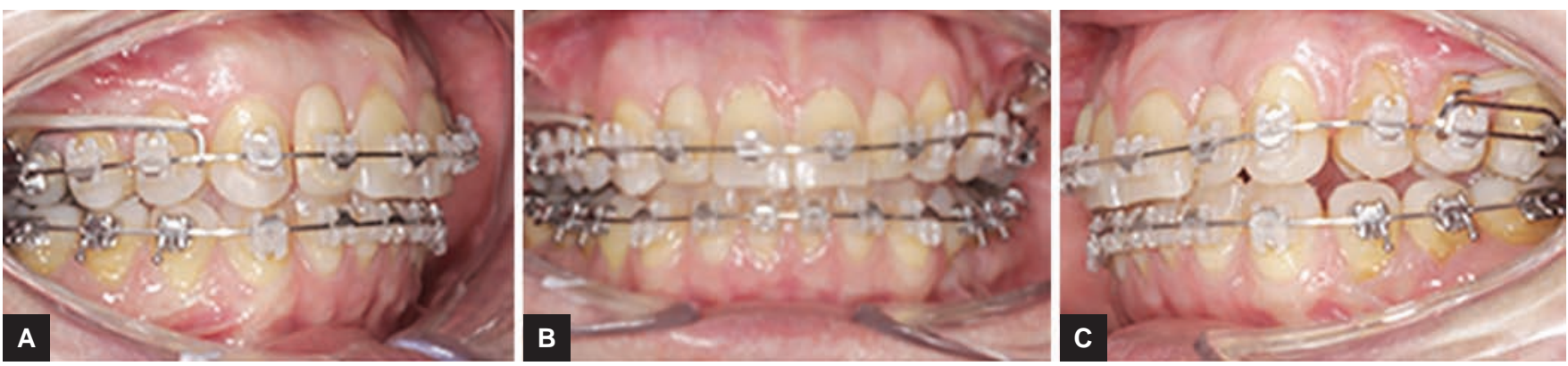

Figs 7A to C: Sliding jigs linking the upper first molar to miniplates to start distal displacement of upper first molars
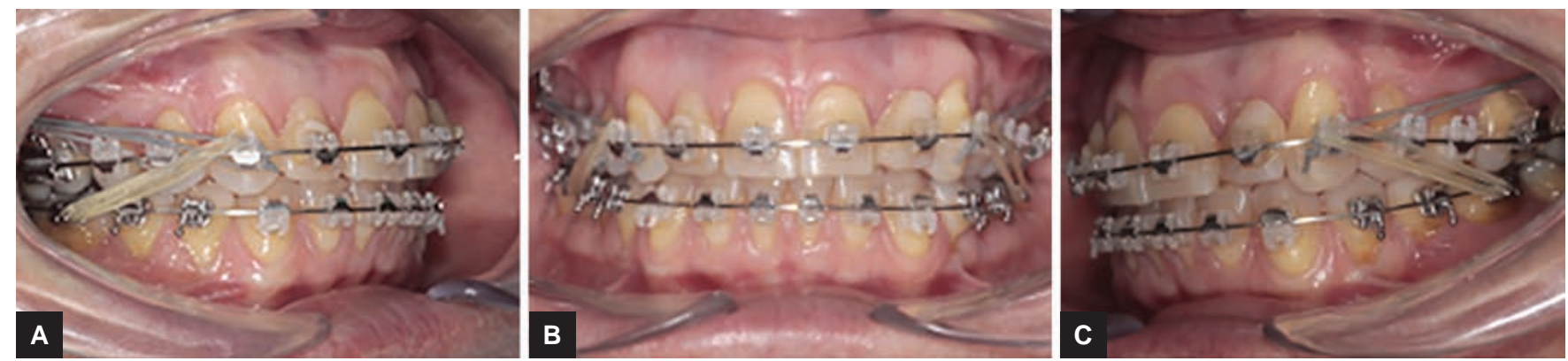

Figs 8A to C: Zing String ${ }^{\mathrm{TM}}$ Elastomeric Thread (TP Ortho) tied from upper cuspids (left and right) to the miniplate associated with nocturnal inter maxillary $1 / 4$ elastics (Morelli-4 oz) 

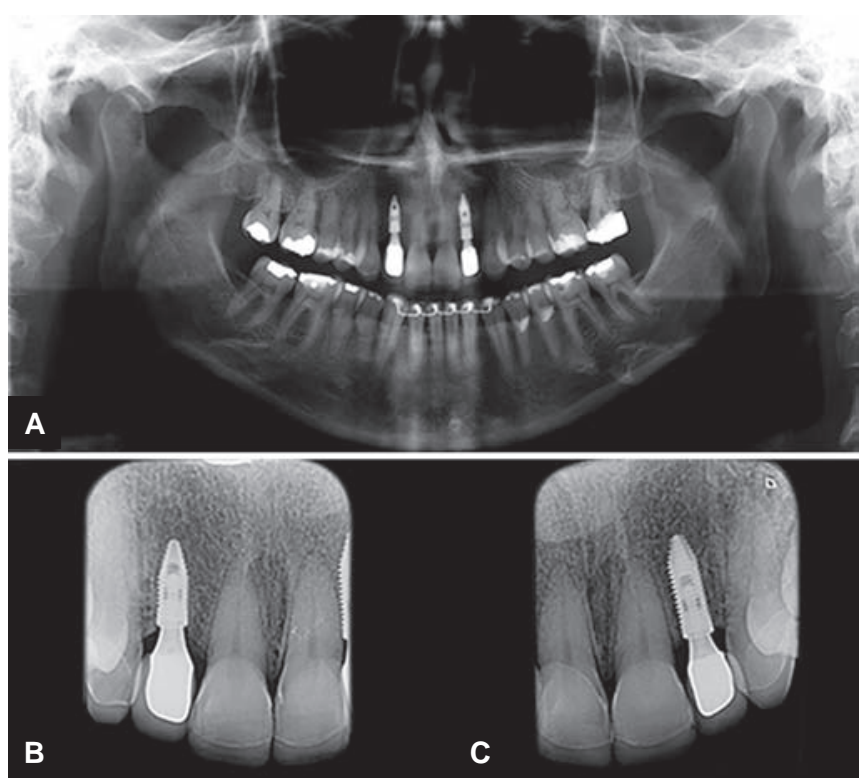

Figs 9A to C: Panoramic (A) and periapical radiographs; (B and C) after treatment, showing implants of both upper lateral incisors

insertion without bone grafting. A3.5-mm diameter Cone Morse implant (Neodent, Curitiba, Brazil) was placed on both sides (Fig. 9).

After complete distalization, slight re-anatomization procedures of maxillary central incisors and canines were requested to adjust the proportion of the dental crowns.
About 19 months later, the fixed appliance was removed, and a minor occlusal adjustment was performed (Figs 10 and 11). A fixed retainer on mandibular anterior teeth and a removable retainer in the maxillary arch (Wraparound) were used.

\section{Treatment Results}

Class I occlusion and ideal conditions of implants and crowns were achieved after treatment (Fig. 12). In addition, we were able to obtain canine guidance during lateral excursion and proper anterior guidance during protrusion. The patient profile was maintained, and overall esthetics improved (Fig. 13).

Cephalometric analysis showed molar distalization of approximately $3.5 \mathrm{~mm}$ from the pterygoid vertical to the distal aspect of upper molars (U6-PTV) (Fig. 14 and Table 1).

The use of miniplates as anchorage provided the posterior teeth of the upper arch to be distalized without reactive movement; therefore, there were no biomechanic side effects, and patient compliance was not essential for treatment success.

\section{DISCUSSION}

Although space closing may be selected as a treatment option, considering it reduces costs on implants and
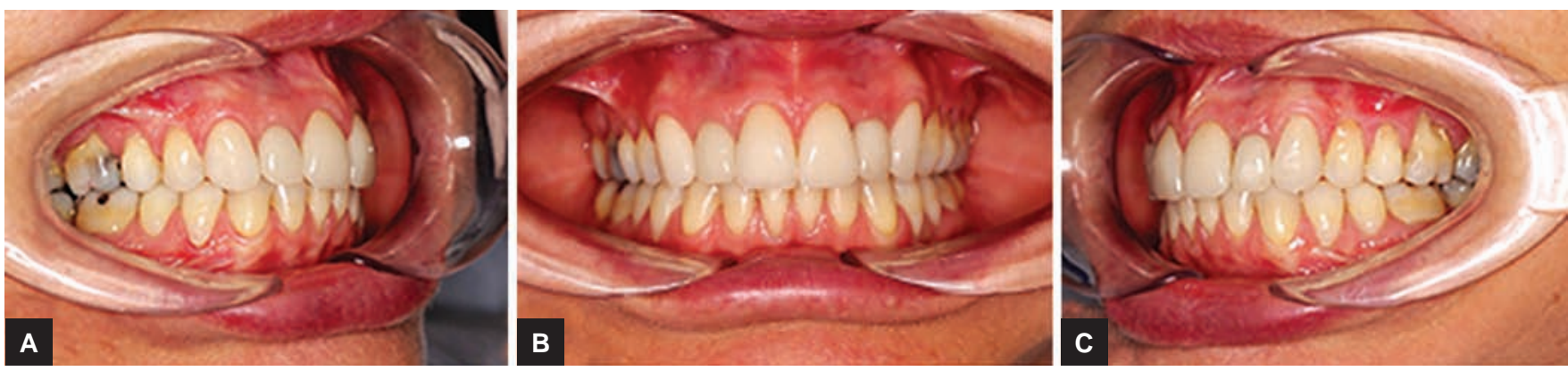

Figs $10 \mathrm{~A}$ to C: Intraoral images after treatment: (A) Right side; (B) front; and (C) left side
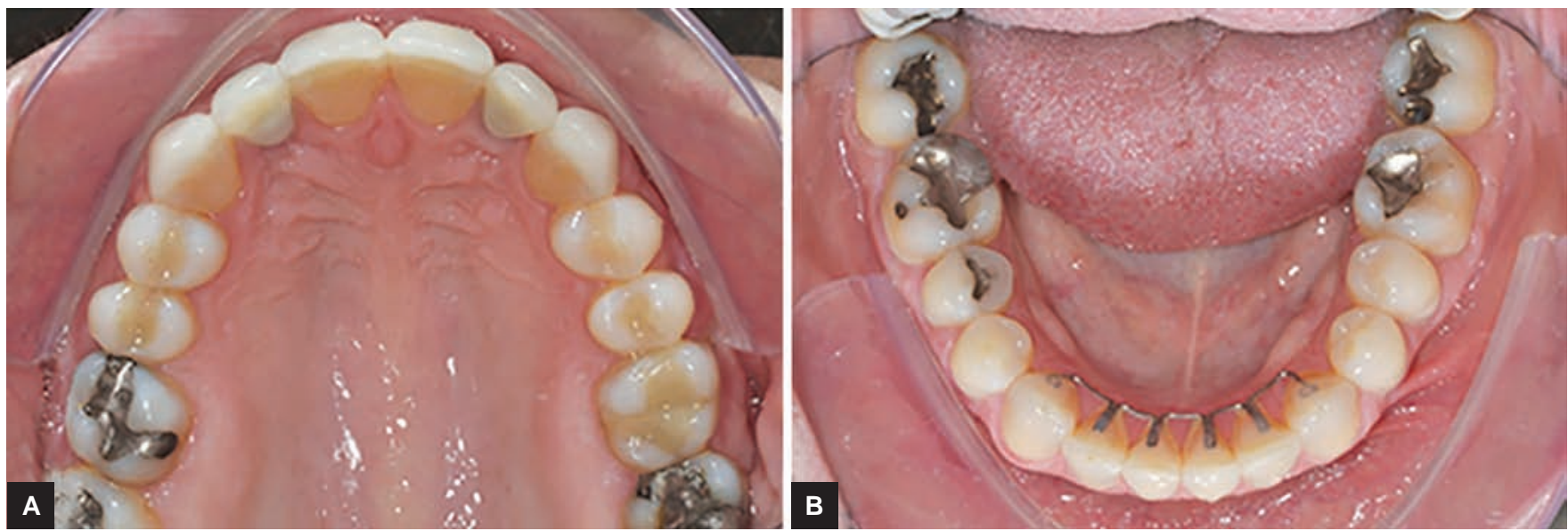

Figs 11A and B: Intraoral images after treatment: (A) Upper occlusal; and (B) lower occlusal 

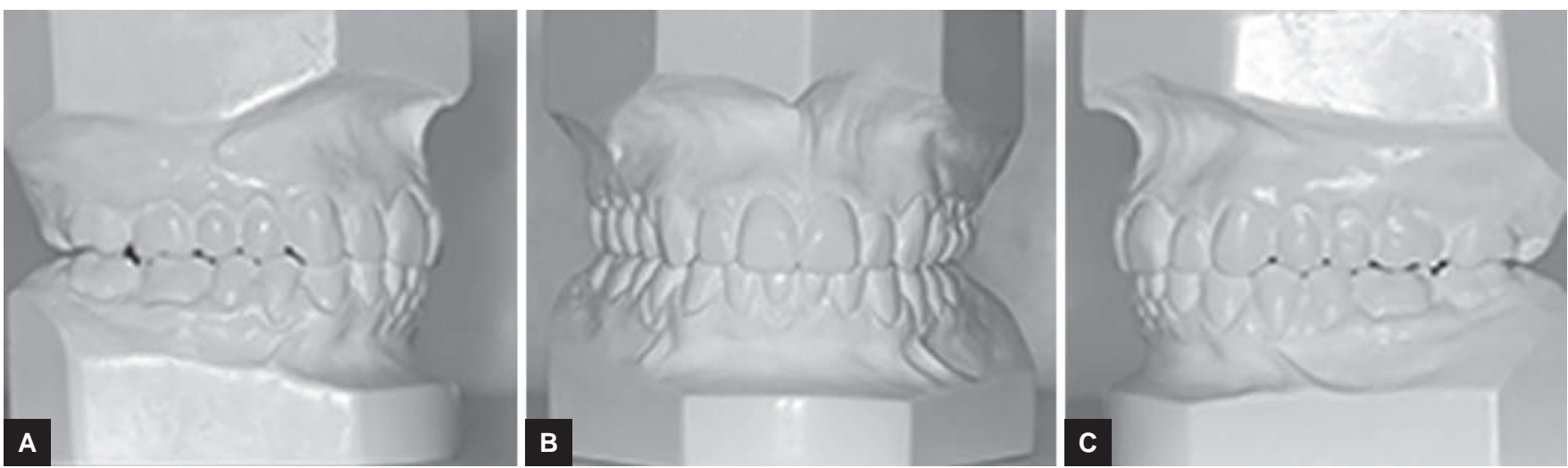

Figs $12 \mathrm{~A}$ to $\mathrm{C}$ : Plaster casts after treatment. Note the distal displacement of upper first molars
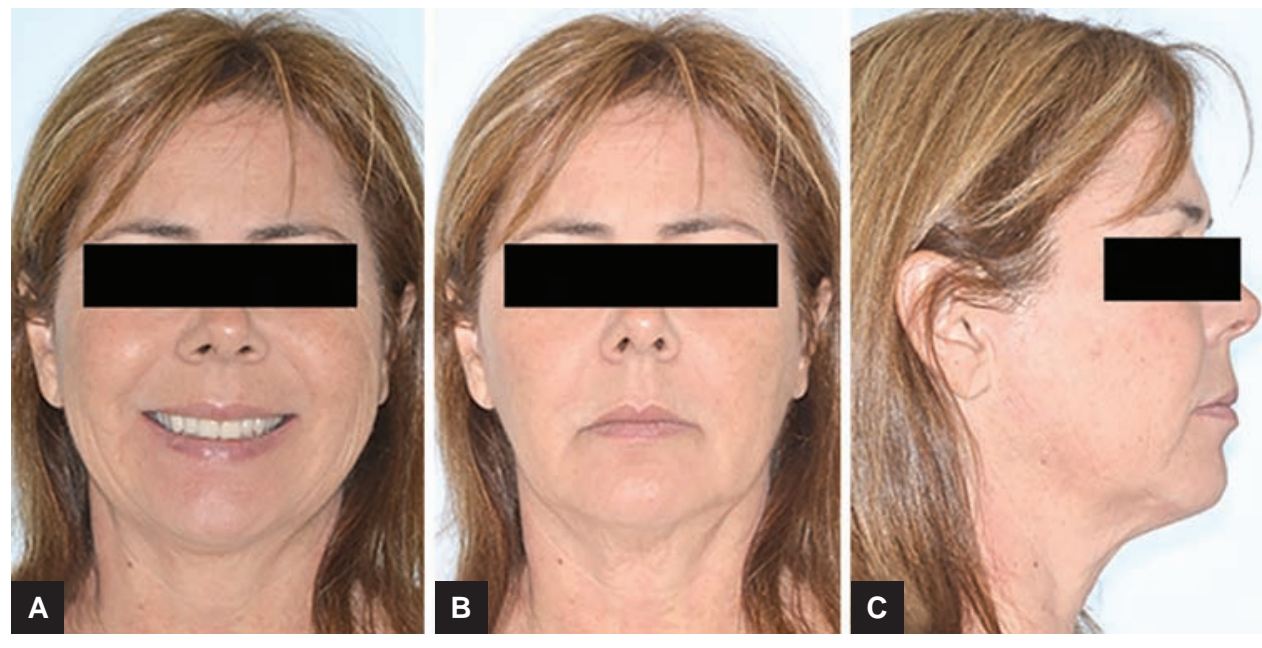

Figs 13A to C: Extraoral images after treatment: (A) Smiling; (B) front; and (C) profile

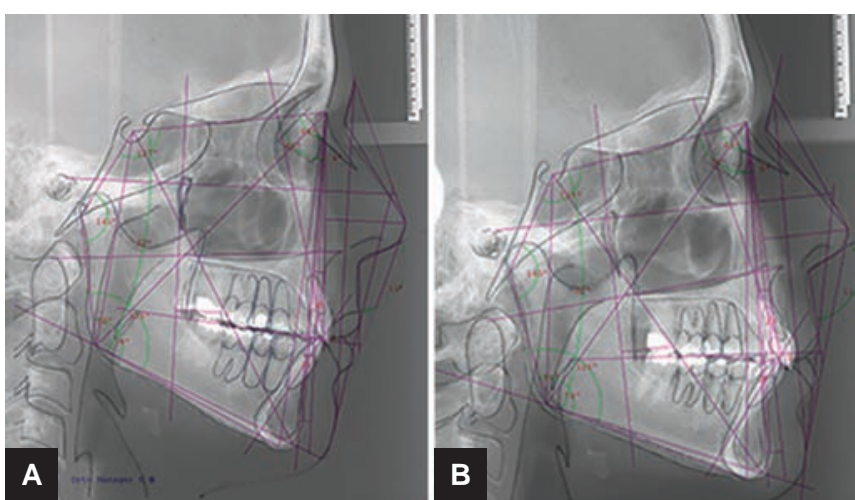

Figs 14A and B: (A) Cephalometric analyses before treatment; and $(B)$ Cephalometric analyses after treatment

prosthesis, there is much to account for when the treatment option is space closing. Such considerations include size difference between the canine and premolar crowns, which may create a poor esthetic balance; color difference between the involved teeth (canines are more yellowish); crown torque differences; functional occlusion at the end of treatment; and risk of relapse after retention. Therefore, the careful detailing throughout the orthodontic progress and finishing stages to achieve optimal positioning and crown torque of all teeth, coupled with new techniques and materials adapted from esthetic dentistry, may restore natural tooth shapes and sizes, provide normal gingival contours around the teeth, and secure an optimally functioning occlusion with cuspid guidance. ${ }^{9}$ The treatment of space opening with implants is considered an innovative and more conservative approach, considering it maintains the canine in its natural position and ideal intercuspation, preserving the morphological features of canines and first premolars, and showing predictable long-term results. ${ }^{2,3,10}$ The factors favoring space closure include the tendency toward maxillary crowding in a patient with good profile, cuspids of similar sizes, dentoalveolar protrusion, class II malocclusion, and mandibular crowding or protrusion. On the contrary, the factors favoring space opening are no malocclusion, space in the maxillary dentition, class III malocclusion and a retrognathic profile, and a large size difference between canines and premolars.?

Space opening may require the same treatment duration as space closing, as seen in the patient from this study, whereas the canine may erupt close to the central incisor when lateral incisors are congenitally missing. However, this may in fact represent an ideal situation due 
to the maintenance of alveolar bone volume at the lateral incisor area. In addition, during the distal movement of the canine, root movement favors the enlargement of the alveolar ridge to the mesial surfaces of the canine through the stretching of the periodontal ligament, avoiding the eventual need for grafts. ${ }^{11}$

The complete diagnosis is performed by gathering medical and dental history information with clinical and radiographic examinations, and more recently, conebeam computed tomography scans have been a very important tool for implant assessment and planning., ${ }^{3,5}$ Therefore, after careful examination, we opted for maxillary lateral incisor implants due to high success rates in the anterior region and the preservation of integrity of adjacent teeth. ${ }^{12}$

To facilitate distal movement of posterior teeth, a self-ligating bracket system was chosen to provide lower frictional resistance when compared with conventional systems connected by elastomeric or metal ligatures. In a reduced friction environment between bracket and wire, the use of lighter forces on these systems would be possible, which potentially could generate less discomfort for the patient, faster tooth movement, and less risk of undesired movements. ${ }^{13}$

Although miniplate placement/removal surgery requires the elevation of a flap, this is considered an easy and relatively short surgical procedure that may be typically performed under local anesthesia without complications, and it may be considered a safe and effective adjunct for the orthodontic treatment. ${ }^{14}$ A 3-hole Y-type titanium miniplate was placed in the buccal cortical plate of the maxilla and secured by three monocortical screws above the dental roots. Although many different types of miniplates are available, the miniplate with a $Y$ design offers an easy way to bend and fit over the zygomatic crest. The authors of this study had previous failures using miniplates retained with two screws in the maxilla. Considering the thin monocortical bone in this area, three screws were used to prevent instability or miniplate loss. No mobility of miniplates was seen at any time during activation. Miniplate stability on this site has been evidenced by several studies in the literature. ${ }^{14,15}$ A 150-g force was applied after 3 weeks, for studies have shown that early loading at 3 weeks after insertion was the most significant factor predicting miniplate failures. ${ }^{16}$

A prospective study showed the effects of maxillary molar distalization in patients treated with skeletal anchorage miniplates and found that upper molars were moved distally $3.27 \pm 1.75 \mathrm{~mm}$ on average, ${ }^{17}$ which agrees with this case report.

Furthermore, the patient opted to rehabilitate her worn teeth with porcelain veneer blades, canine to canine for final esthetic and rehabilitation, and to achieve ideal esthetics of the lateral incisor crown with all the anterior teeth.

Several studies have shown advantages and disadvantages from both treatments, either closing spaces ${ }^{7,18}$ or opening spaces, ${ }^{19}$ with similar satisfactory and functional results. ${ }^{1,7}$ Although there is much controversy in the literature, ${ }^{2,4,20}$ long-term follow-up studies are still needed to determine the stability of such treatments. However, it is safe to state that proper multidisciplinary diagnosis and treatment planning are imperative to define treatment options to provide the best result for each patient. ${ }^{2}$ In practice, treatment protocol should be performed with extreme caution, based on professional clinical skills and experience, clinical conditions of each patient, and patient expectations. $^{7,21}$

\section{CONCLUSION}

When indicated, space opening may provide excellent esthetics and functional results, and even more predictable results when skeletal anchorage miniplates are used to distalize all posterior teeth, combined with self-ligating brackets, which leads to faster movement, controlled mechanics, and less dependence on patient compliance.

\section{CLINICAL SIGNIFICANCE}

Considering the high level of esthetic and functional compromise caused by dental agenesis, the technique hereby described represents a viable mechanic alternative within orthodontic possibilities. Moreover, the technique used in the present case report is favorable for not requiring patient compliance.

\section{REFERENCES}

1. De Marchi LM, Pini NI, Hayacibara RM, Silva RS, Pascotto RC. Congenitally missing maxillary lateral incisors: functional and periodontal aspects in patients treated with implants or space closure and tooth re-contouring. Open Dent J 2012 Dec;6:248-254.

2. Pini NI, Marchi LM, Pascotto RC. Congenitally missing maxillary lateral incisors: update on the functional and esthetic parameters of patients treated with implants or space closure and teeth recon touring. Open Dent J 2015 Jan;8:289-294.

3. Kavadia S, Papadiochou S, Papadiochos I, Zafiriadis L. Agenesis of maxillary lateral incisors: a global overview of the clinical problem. Orthodontics (Chic.) 2011 Winter; 12(4):296-317.

4. Kokich VO Jr, Kinzer GA. Managing congenitally missing lateral incisors. Part I: canine substitution. J Esthet Restor Dent 2005 Jan;17(1):5-10

5. de Almeida RR, Morandini AC, de Almeida-Pedrin RR, de Almeida MR, Castro RC, Insabralde NM. A multidisciplinary treatment of congenitally missing maxillary lateral incisors: a 14-year follow-up case report. J Appl Oral Sci 2014 Sep-Oct;22(5):465-471. 
6. Rosa M, Zachrisson BU. Integrating esthetic dentistry and space closure in patients with missing maxillary lateral incisors. J Clin Orthod 2001 Apr;35(4):221-234.

7. Rosa M, Lucchi P, Ferrari S, Zachrisson BU, Caprioglio A. Congenitally missing maxillary lateral incisors: long-term periodontal and functional evaluation after orthodontic space closure with first premolar intrusion and canine extrusion. Am J Orthod Dentofacial Orthop 2016 Mar;149(3):339-348.

8. De Clerck EE, Swennen GR. Success rate of miniplate anchorage for bone anchored maxillary protraction. Angle Orthod 2011 Nov;81(6):1010-1013.

9. Janson G, Valarelli DP, Valarelli FP, de Freitas MR, Pinzan A. Atypical extraction of maxillary central incisors. Am J Orthod Dentofacial Orthop 2010 Oct;138(4):510-517.

10. NováckováS, Marek I, Kamínek M. Orthodontic tooth movement: bone formation and its stability over time. Am J Orthod Dentofacial Orthop 2011 Jan;139(1):37-43.

11. de Avila ÉD, de Molon RS, de Assis Mollo F Jr, de Barros LA, Capelozza Filho L, de Almeida Cardoso M, Cirelli JA. Multidisciplinary approach for the aesthetic treatment of maxillary lateral incisors agenesis: thinking about implants? Oral Surg Oral Med Oral Pathol Oral Radiol 2012 Nov;114(5): e22-e28.

12. Mayer TM, Hawley CE, Gunsolley JC, Feldman S. The singletooth implant: a viable alternative for single-tooth replacement. J Periodontol 2002 Jul;73(7):687-693.

13. Damon DH. The rationale, evolution and clinical application of the self-ligating bracket. Clin Orthod Res 1998 Aug;1(1):52-61.
14. Cornelis MA, Scheffler NR, Mahy P, Siciliano S, De Clerck HJ, Tulloch JF. Modified miniplates for temporary skeletal anchorage in orthodontics: placement and removal surgeries. J Oral Maxillofac Surg 2008 Jul;66(7):1439-1445.

15. Sherwood KH, Burch JG, Thompson WJ. Closing anterior open bites by intruding molars with titanium miniplate anchorage. Am J Orthod Dentofacial Orthop 2002 Dec;122(6):593-600.

16. Chen YJ, Chang HH, Lin HY, Lai EH, Hung HC, Yao CC. Stability of miniplates and miniscrews used for orthodontic anchorage: experience with 492 temporary anchorage devices. Clin Oral Implants Res 2008 Nov;19(11):1188-1196.

17. Cornelis MA, De Clerck HJ. Maxillary molar distalization with miniplates assessed on digital models: a prospective clinical trial. Am J Orthod Dentofacial Orthop 2007 Sep;132(3):373-377.

18. Pini NP, De-Marchi LM, Gribel BF, Pascotto RC. Digital analysis of anterior dental esthetic parameters in patients with bilateral maxillary lateral incisor agenesis. J Esthet Restor Dent 2013 Jun;25(3):189-200.

19. Mangano C, Levrini L, Mangano A, Mangano F, Macchi A, Caprioglio A. Esthetic evaluation of implants placed after orthodontic treatment in patients with congenitally missing lateral incisors. J Esthet Restor Dent 2014 Jan-Feb;26(1):61-71.

20. Andrade DC, Loureiro CA, Araújo VE, Riera R, Atallah AN. Treatment for agenesis of maxillary lateral incisors: a systematic review. Orthod Craniofac Res 2013 Aug;16(3):129-136.

21. Antonarakis GS, Prevezanos P, Gavric J, Christou P. Agenesis of maxillary lateral incisor and tooth replacement: cost-effectiveness of different treatment alternatives. Int J Prosthodont 2014 May-Jun;27(3):257-263. 